\title{
Binge eating in patients with polycystic ovary syndrome: prevalence, causes, and management strategies
}

This article was published in the following Dove Press journal:

Neuropsychiatric Disease and Treatment

\author{
Isabel Krug' \\ Sarah Giles' \\ Chiara Paganini ${ }^{2,3}$ \\ 'Melbourne School of Psychological \\ Sciences, The University of Melbourne, \\ Melbourne, VIC, Australia; ${ }^{2}$ Faculty of \\ Health, University of Tasmania, Hobart, \\ Tasmania, Australia; ${ }^{3}$ Faculty of Health, \\ Federation University, Ballarat, VIC, \\ Australia
}

\begin{abstract}
Emerging evidence suggests that disordered eating, particularly binge-eating symptomatology, is overrepresented within Polycystic Ovary Syndrome (PCOS) populations. This comorbidity presents a clinical dilemma as current treatment approaches for PCOS emphasize the importance of weight management, diet, exercise, and the potential for harm of such treatment approaches in PCOS patients with comorbid disordered eating. However, limited research has assessed the occurrence of binge eating and disordered eating in PCOS patients. Consequently, little is known about the prevalence of binge eating in PCOS, and the possible etiological processes to explain this comorbidity remain poorly understood. Given the paucity of research on this topic, the aims of this narrative review are fourfold: 1) to outline the main symptoms of PCOS and binge eating; 2) to provide an overview of the prevalence of binge eating in PCOS; 3) to outline possible etiological factors for the comorbidity between PCOS and binge eating; and 4) to provide an overview of management strategies of binge eating in PCOS.
\end{abstract}

Keywords: PCOS, eating disorders, binge eating, prevalence, risk factors, management

\section{Introduction}

Polycystic Ovary Syndrome (PCOS) is the most common endocrine condition in women of reproductive age, occurring in approximately $15-20 \%$ of the women. ${ }^{1,2}$ PCOS is diagnosed by the presence of 2 of 3 the following criteria: high levels of androgens (male sex hormones), missed or irregular periods, and various small cysts (fluid-filled sacs in the ovaries). ${ }^{3}$ Many established comorbidities have been associated with PCOS including an increased risk for obesity, type II diabetes, cardiovascular diseases, ${ }^{4}$ infertility $^{5}$ and mental disorders such as anxiety, depression, ${ }^{6}$ and eating disorders (EDs). ${ }^{7}$ EDs characterized by binge eating behaviors, such as bulimia nervosa (BN) and binge eating disorder (BED) appear to be the most prevalent disorders within PCOS populations; suggesting that binge-eating symptomatology may be particularly relevant within this population. ${ }^{7}$ However, to date, relatively few studies ${ }^{8}$ have assessed the occurrence of binge eating in PCOS patients. Consequently, little is known about the prevalence of binge eating in PCOS, and the etiological processes to account for this comorbidity. This is particularly important given that current treatment guidelines for PCOS emphasize the importance of weight management, dietary interventions, and physical activity, in the management of PCOS and the potential for harm of such treatment approaches for individuals with comorbid disordered eating. ${ }^{9}$ Furthermore,
Correspondence: lsabel Krug School of Psychological Sciences, University of Melbourne, Redmond Barry Building, Level 7, Room 707, North Melbourne, VIC 3010, Australia

$\mathrm{Tel}+61390358551$

Fax +6 I393264774

Email isabel.krug@unimelb.edu.au 
formalized treatment guidelines which address both problem sets simultaneously are currently lacking. Therefore, the aims of the current review are fourfold: 1) to outline the main symptoms of PCOS and binge eating; 2) to provide an overview of the prevalence of binge eating in PCOS; 3) to outline possible etiological factors for the comorbidity between PCOS and binge eating; and 4) to provide an overview of management strategies of binge eating in PCOS.

\section{The main features of PCOS}

Many of the clinical features of PCOS are caused by high levels of androgens resulting in hyperandrogenism. ${ }^{1}$ Hyperandrogenism can lead to many adverse effects in women with this condition, including the growth of ovarian cysts, weight gain, hair growth, especially increased body and facial hair (hirsutism), acne, and hair loss (alopecia) in the central part of the scalp. ${ }^{10,11}$ High levels of androgens affect menstruation and ovulation, and women with PCOS commonly suffer from menstrual irregularities, especially oligomenorrhea (infrequent bleeding), or amenorrhea (no menstrual period) and oligoovulation (infrequent ovulation) or anovulation (absent ovulation). ${ }^{2}$ Additionally, PCOS women present with a range of metabolic features including insulin resistance, type 2 diabetes, and cardiovascular risk factors, and subsequently PCOS is associated with a greater risk of being overweight or obese. ${ }^{12,13}$ Obesity is known to exacerbate all metabolic and reproductive outcomes for women with PCOS, as well as anxiety and depressive symptoms. ${ }^{6,14,15}$

As a chronic disease associated with complex physical and body image related challenges, PCOS is associated with decreased health-related quality of life and emotional well-being. ${ }^{16}$ Additionally, insulin resistance is generally followed by intense carbohydrate cravings, which may promote overeating. ${ }^{11}$ These factors have been strongly associated with the development of psychiatric illnesses including depression, anxiety, and EDs, especially those EDs characterized by binge eating symptomatology. ${ }^{17-19}$

\section{EDs characterized by binge eating behavior}

Binge eating behavior is characterized by 1) eating, in a discrete period of time (eg, within any 2-hr period), an amount of food that is definitely larger than what most people would eat in a similar period of time under similar circumstances; and 2) the binge episode is accompanied by a loss of control over eating (eg, a feeling that one cannot stop eating or control what or how much one is eating). ${ }^{20}$

Various types of EDs, such as BN, Anorexia Nervosa Binge Purging type (AN-BP), BED, and other specified feeding or eating isorder (OSFED) and unspecified feeding eating or disorder, may be characterized by binge eating behaviors. ${ }^{20} \mathrm{BN}$ is estimated to have a prevalence of approximately $1-1.5 \%$ in young adult women. Its main features include recurrent episodes of binges, and compensatory behaviors such as purging behavior, excessive exercise, and/or dieting, which individuals employ to control their shape and weight. ${ }^{20}$ AN-BP is characterized by the same binge-purging criteria as $\mathrm{BN}$, but individuals experiencing this ED diagnosis generally present with a lower body mass index (BMI, $<18.5 \mathrm{~kg} / \mathrm{m}^{2}$ ). Its prevalence estimates are lower than $\mathrm{BN}$, with an estimated occurrence of $0.4 \%{ }^{20}$

Unlike those with BN and AN-BP, individuals with BED do not use compensatory behaviors, such as selfinduced vomiting or over-exercising following binge eating episodes. BED is therefore exclusively characterized by binge eating episodes. It is more common than $\mathrm{BN}$ and AN-BP, with an estimated prevalence of $1.6 \%$ in the general population. ${ }^{20}$ Most individuals with BED are overweight or obese and approximately $50 \%$ of the people diagnosed with BED are clinically obese. ${ }^{21}$ Finally, OSFED may present with many of the symptoms such as AN-BP, BN, or BED, but individuals diagnosed with this disorder do not fulfill the diagnostic criteria for these ED diagnoses. Currently, the DSM- $5^{20}$ outlines five different types of OSFED, which include: atypical AN, BN (of low frequency and/or limited duration), BED (of low frequency and/or limited duration), purging disorder, and night eating syndrome. OSFED is the most common ED diagnosis, and it is estimated that around $30 \%$ of the people, who seek treatment for an ED have OSFED. However, there are currently only very few prevalence studies of OSFED, mainly because of the difficulties in capturing its heterogeneous nature. The few studies reported in the literature have so far shown a prevalence of OSFED of approximately $3-6 \%$ in community samples. ${ }^{22}$

Binge eating is associated with difficulties managing weight, including weight gain, ${ }^{23}$ obesity onset, ${ }^{24}$ and unsuccessful attempts to lose weight. ${ }^{25}$ Binge eating has also been associated with impaired physical health and quality of life. ${ }^{26}$ Moreover, individuals with binge eating often have impaired psychological functioning, including 
high rates of comorbid psychological disorders, such as anxiety and depression, ${ }^{27}$ which as previously outlined, are also common in women with PCOS women. ${ }^{8,19}$ Such impairments in psychological function may be partially attributed to the binge eating symptomatology, since individuals typically experience shame and distress as a result of their eating patterns. ${ }^{28,29}$ EDs characterized by bingeing symptoms, particularly BED, have been associated with similar physical comorbidities evident in PCOS populations, including an increased risk of medical complications associated with obesity, such as type II diabetes, cardiovascular disorders, and fertility issues. ${ }^{30,31}$ These findings demonstrate significant commonalities in psychological and physical comorbidities present in individuals with PCOS and binge eating behaviors.

\section{Method for review}

Despite research indicating that women with PCOS are more likely to experience ED pathology, particularly binge eating behaviors, a paucity of research has explored the prevalence of these behaviors in women with PCOS. A systematic review was not conducted to explore this question, rather the literature was narratively reviewed based on clinical expertise. In this narrative review, collated research findings, which examined the presence of disordered eating behaviors and/or ED diagnoses, in women with PCOS are presented. We assembled relevant research findings without imposing specific inclusion/exclusion criteria (eg, criteria for PCOS diagnosis, inclusion of a comparison group, or ED assessment method). Subsequently, this review extends existing literature, by including studies beyond those only diagnosed using the PCOS Rotterdam criteria. ${ }^{3}$ The Rotterdam criteria define PCOS by the presence of at least two out of three criteria: oligo-anovulation, clinical, and/or biochemical hyperandrogenism and polycystic ovaries ( $\geq 12$ follicles measuring $2-9 \mathrm{~mm}$ in diameter or ovarian volume $0.10 \mathrm{~mL}$ in at least one ovary). ${ }^{3}$ While these criteria are endorsed in treatment guidelines for diagnosing PCOS in adults, ${ }^{32}$ the development of these criteria was based on expert meetings and not on evidence-based treatment guidance. ${ }^{33}$ Additionally, literature has shown that overall the diagnosis of PCOS is difficult, as the exhibiting signs and symptoms are diverse, depending on the populations assessed, the degree of obesity, and life phase of the women experiencing this disorder. ${ }^{32}$ Therefore, by broadening our inclusion criteria, we aim to examine the relationship between PCOS and binge eating behaviors within diverse PCOS phenotype groups.

\section{Prevalence of binge eating in PCOS patients}

The results of the narrative review are presented in Table 1. Eight of the studies presented ${ }^{8,15,34-39}$ were included in a recent meta-analysis, ${ }^{7}$ which assessed the increased likelihood of experiencing ED symptoms in PCOS populations in comparison to healthy female controls. The authors of this review performed a systematic review and metaanalysis of studies that assessed women with well-defined PCOS, according to either contemporary Rotterdam criteria $^{3}$ or NIH criteria and controls without PCOS. Furthermore, the selected studies employed validated ED screening tools to assess abnormal ED scores and/or prevalence of specific ED diagnoses. Results ${ }^{8,15,34-39}$ included 470 women with PCOS and 390 controls, who met inclusion criteria for the systematic review.

A meta-analysis on seven of these studies revealed that women with PCOS had over three times the odds of having abnormal ED scores (OR 3.05; 95\%CI 1.33, 6.99 four studies) as well as being diagnosed with an ED (OR 3.87; 95\% CI 1.43, 10.49), when compared to women without PCOS. However, there was not enough power to statistically assess which exact ED subtype this increased risk was attributable to. The authors did note though that the PCOS group revealed a significantly higher BMI than the control sample. ${ }^{20}$ This is significant as obese individuals with BED, and non-obese BED groups differ in their drive for thinness, frequency of objective binge eating episodes, and concern about weight. $^{40,41}$ Therefore, BMI differences are likely to influence binge eating behaviors, and BMI differences rather than PCOS status, may account for some variation in binge-eating symptomatology. The remaining studies included in the narrative review assessed ED behaviors in PCOS women $^{17,18,42-52}$ without imposing inclusion criteria (eg, PCOS diagnosed according to Rotterdam criteria ${ }^{3}$ or NIH criteria, controls without PCOS, and type of ED screening tools). The studies examining clinically significant disordered eating symptoms reported a prevalence between $6.8 \%$ and $58 \%$ for binge eating symptoms, ${ }^{17,46,48,51}$ and between $2.3 \%$ and $33.33 \%^{17,42,49}$ for BN symptoms. The prevalence of a diagnosed ED varied between diagnostic categories, for $\mathrm{AN}$ estimates, ranged between $0.0 \%$ and $1.3 \%,{ }^{17,18,44,50}$ for $\mathrm{BN}$ between $0.0 \%$ and $12.6 \%,{ }^{17,18,42,44,50}$ for BED between $4 \%$ and $15 \%,{ }^{17,44,50,52}$ and one study reported a prevalence of $22.5 \%$ for EDNOS. ${ }^{50}$

Two studies ${ }^{43,47}$ assessed dietary patterns in women with PCOS. Results showed that PCOS women had higher 


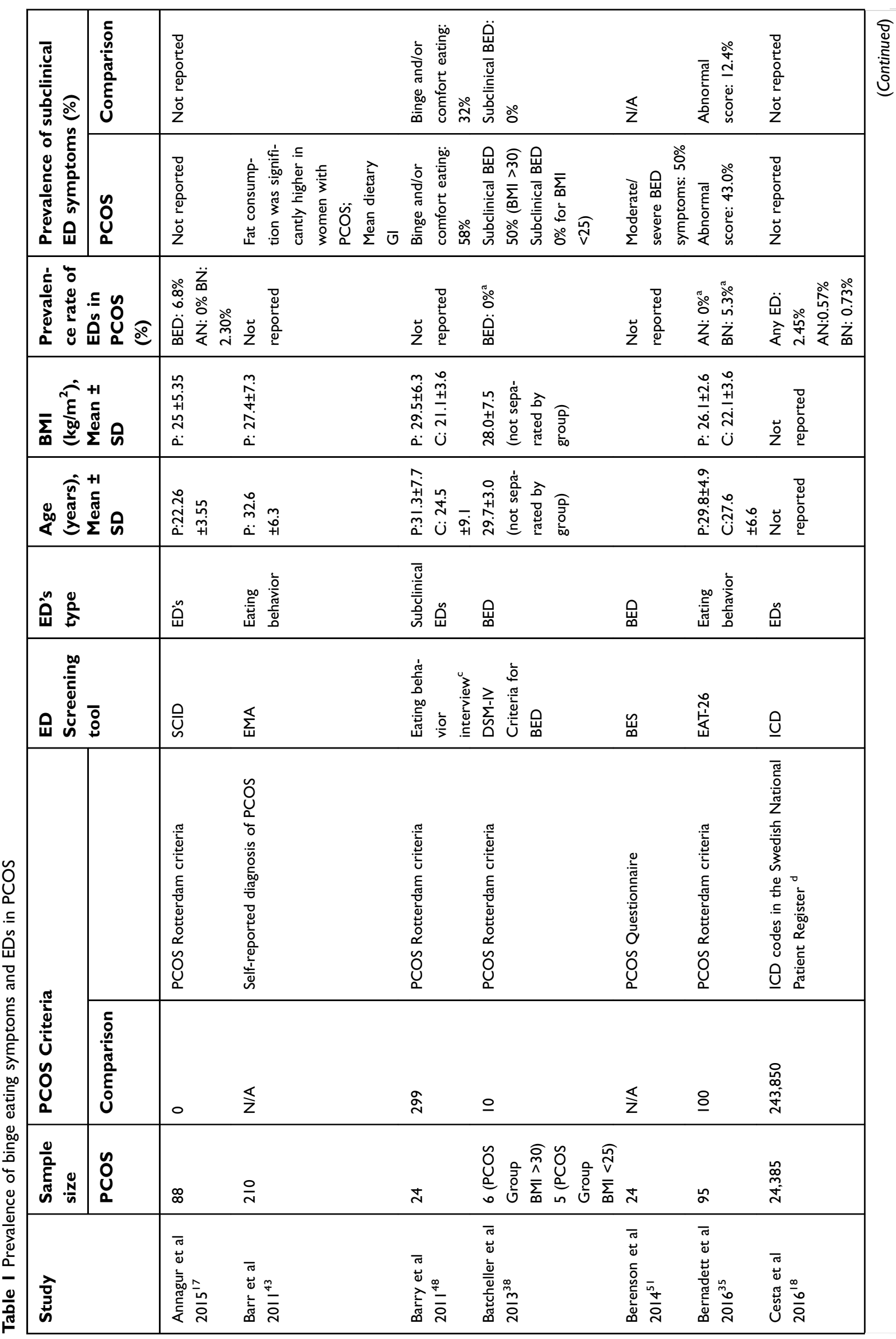




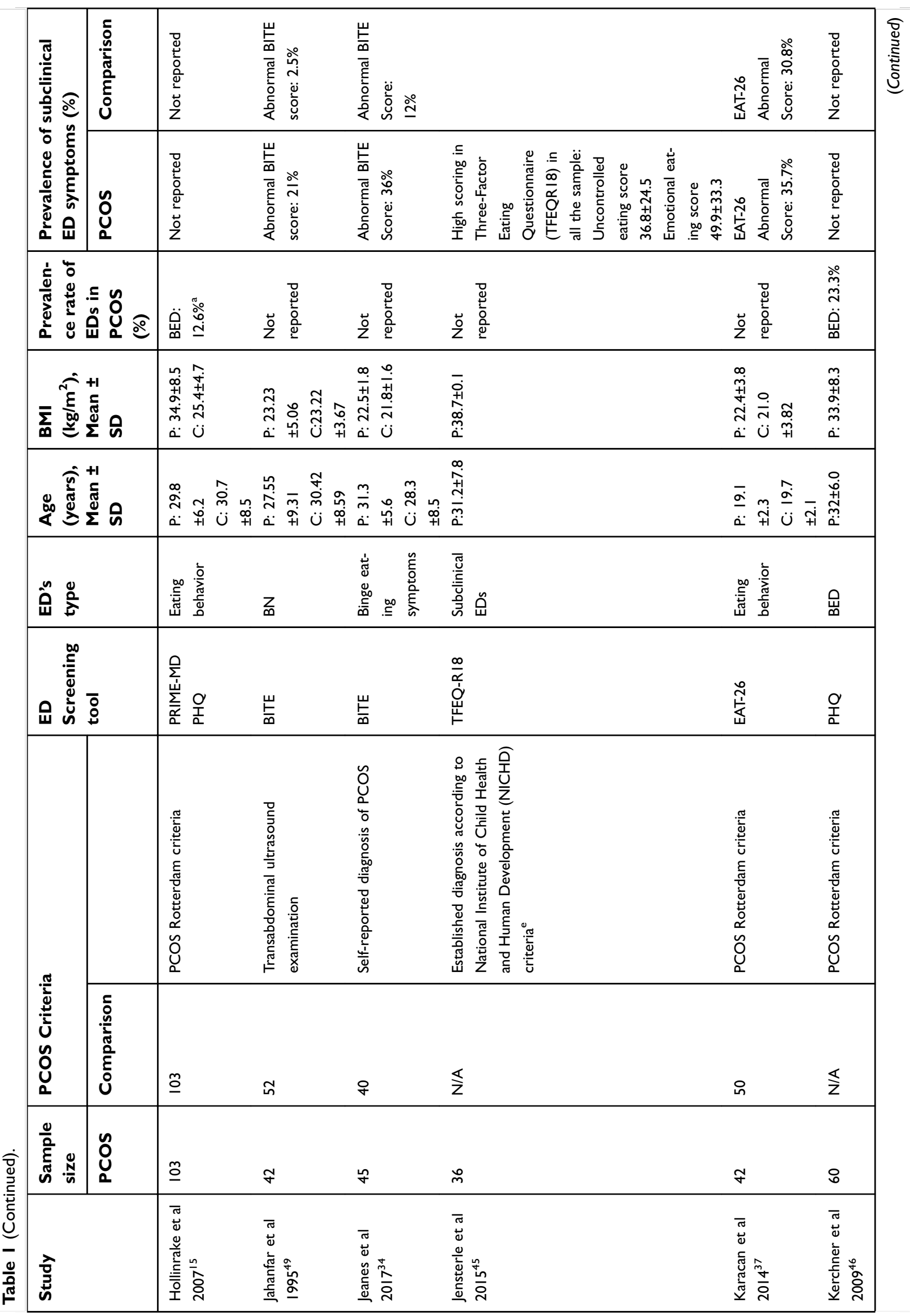




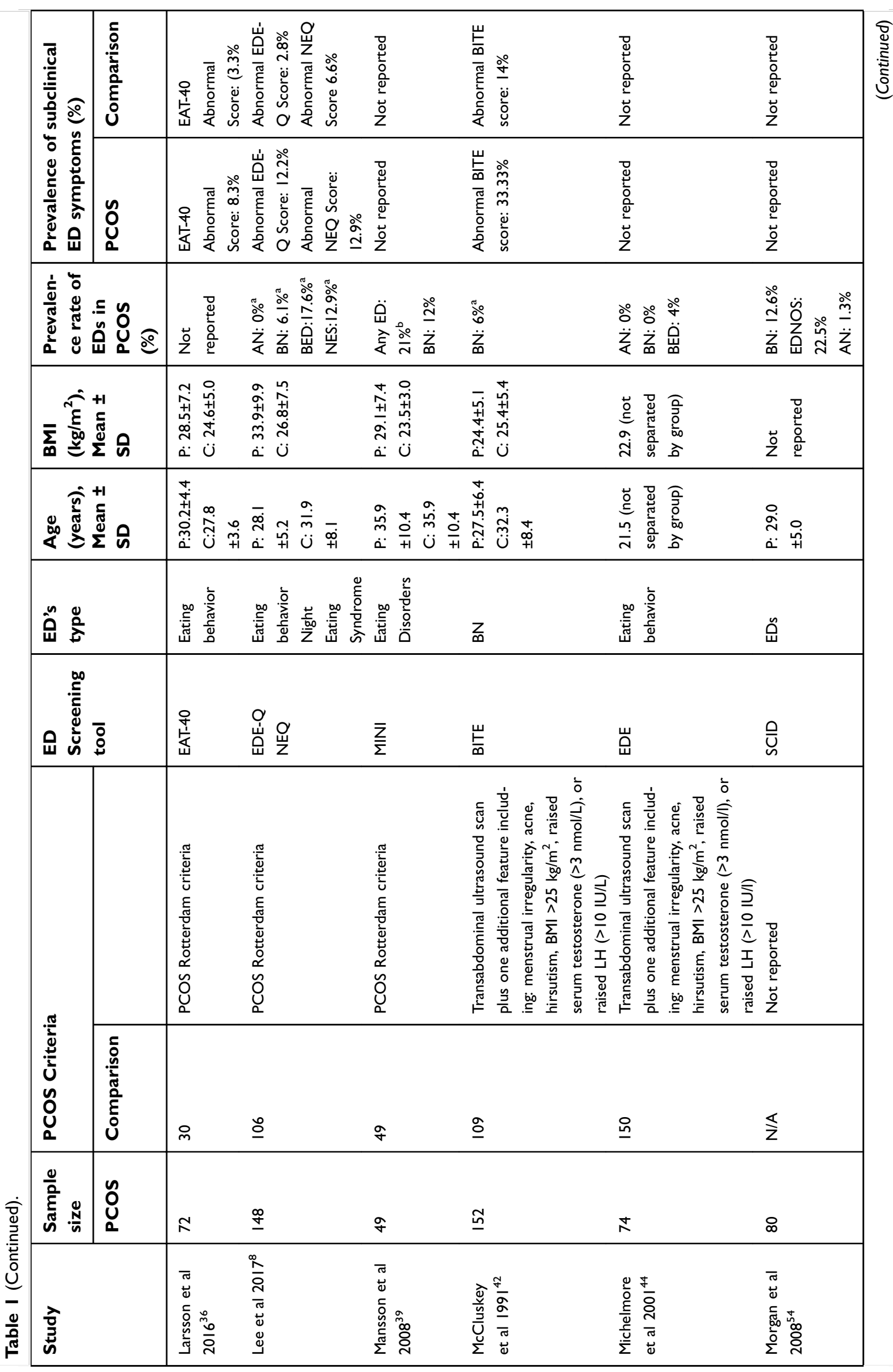




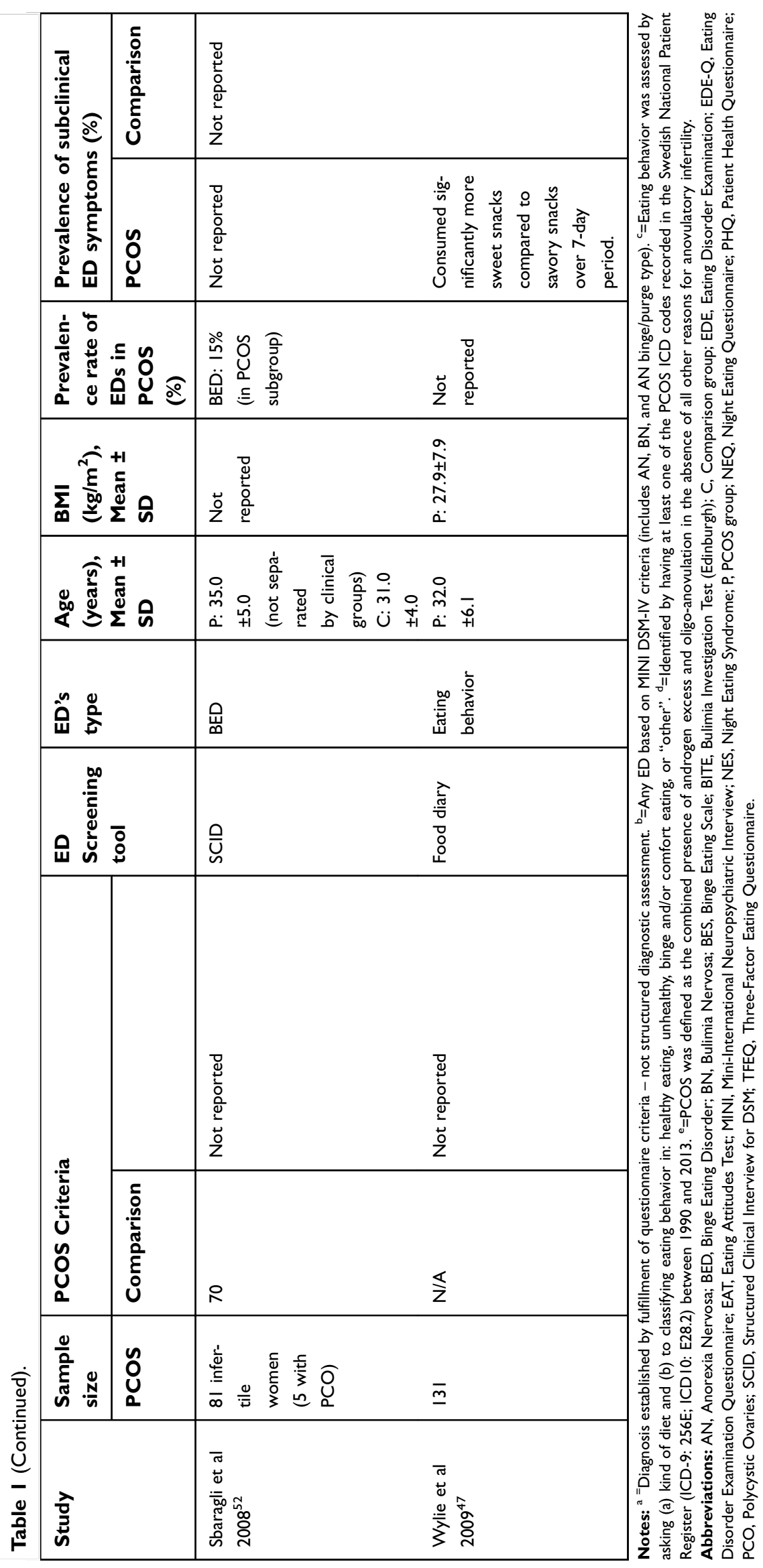


overall energy intake than national averages, consumed significantly more sweet than savory snacks, and that BMI was negatively associated with eating frequency,. These findings suggest that PCOS women's overall energy intake is higher than average, and is consumed in fewer episodes of eating.

The overall prevalence of any ED presented in the current review ranged from $0 \%$ to $22.5 \%$, while the prevalence of clinically significant disordered eating was generally higher, ranging from $8.3 \%$ to $58 \%$. This is significant, as many women will report disordered eating symptoms, that are associated with distress and impairment, which may not fulfill diagnostic criteria for a specific ED diagnosis. ${ }^{53}$

Regarding the assessment of disordered eating symptoms, the studies included in the review varied considerably in their assessment. The majority of these studies used selfreport questionnaires, such as the Eating Attitudes Test- $26^{52}$ $\mathrm{EAT}^{55} \mathrm{eg}^{31,32}$ ) or the Bulimia Investigation $\mathrm{Test}^{56}$, Edinburgh $^{53}$ (BITE eg, ${ }^{30,45}$ ) to assess ED symptoms. Studies which employed self-report measures to assess disordered eating $3,8,30-33,37,40,44,45,49$ reported clinically elevated ED symptoms in women with PCOS.

While these studies provide evidence that ED symptoms are elevated in PCOS populations, reliable inferences about the prevalence and nature of ED diagnoses in this population are difficult to infer from self-report measures. Interviewbased methods for diagnosing EDs, such as the $\mathrm{SCID}^{57}$ or the Eating Disorder Examination ${ }^{58}$, may be better placed to reliably characterize the prevalence of EDs in women with PCOS. However, these measures were only used in a small proportion of the studies included (eg, 15,38,46,50). Furthermore, several studies did not include a control group (eg, ${ }^{17,43,45-47,50}$ ). Most studies (eg, ${ }^{17,38,48}$ ) used the PCOS Rotterdam criteria to establish a diagnosis, while other studies used self-report measures to diagnose PCOS (eg, ${ }^{34,43,51}$ ), and only a few studies employed ultrasound to establish a PCOS diagnosis $\left(\mathrm{eg}^{42,44,49}\right)$. There are also a few studies $\left(\mathrm{eg},{ }^{47,50,52}\right)$ that did not provide any information on how PCOS was assessed. Consequently, caution should be exercised when interpreting the findings of these studies.

In summary, the results of the review demonstrate that EDs, particularly those characterized by binge-eating, are prevalent in PCOS populations, and that a significant proportion of women with PCOS report clinically significant disordered eating symptoms that may not necessarily meet the threshold for an ED diagnosis. The variability in prevalence estimates of disordered eating in PCOS may be attributable to the measures employed to assess disordered eating. Diagnostic overshadowing of PCOS symptoms may also impede a reliable diagnosis, and concealment and shame of disordered eating symptoms, or the health care professionals' dearth of knowledge and skill sets in identifying and treating ED symptoms may also contribute to variable prevalence estimates. ${ }^{17,59}$

\section{What are the connections between PCOS and binge eating behavior?}

Despite the association between PCOS and binge eating, an etiological link has never been satisfactorily explained. ${ }^{19}$ However, the development of binge eating behavior among women with PCOS is unsurprising, given the overlap in symptoms such as difficulty losing weight, irregular menstrual cycles, infertility, and hirsutism. ${ }^{1,19,34}$ The connections between PCOS and binge eating are most likely numerous and complex. In the subsequent paragraphs, we will, therefore, provide a distinction between the most prominent metabolic, hormonal, and psychological factors, that may explain the relationship between the two disorders.

\section{Hormonal factors - hyperandrogenism}

Research has found that hormonal and menstrual irregularities confer vulnerability to both $\operatorname{PCOS}^{1,10,11}$ and binge eating behavior. ${ }^{60,61}$ Androgens, including testosterone, are present in both sexes, however, the levels are generally lower in females than males. A prominent feature of PCOS is elevated concentrations of testosterone, which account for numerous of the PCOS related symptoms, including hirsutism, acne, scalp hair loss, irregular menses, and diminished fertility. ${ }^{911,62}$ Hyperandrogenism is implicated in $\operatorname{PCOS}^{9}$ and binge eating behavior ${ }^{63,64}$ and has frequently been linked to the causation of menstrual irregularities, including amenorrhea, and a delay and/or lack of ovulation. ${ }^{65,66}$ These menstrual irregularities may lead to increased hunger and psychological distress, potentially resulting in an increase in binge eating frequency. ${ }^{61,67}$

Another potential hypothesis for the relationship between PCOS and binge eating behavior may be that hyperandrogenism influences the occurrence of bulimic behavior. As previously outlined, high androgen levels in women have been found to stimulate appetite ${ }^{64,68}$ and increase anxious and depressed mood states. ${ }^{69,70}$ Therefore, high androgen levels may confer risk for binge eating behaviors, as binge eating episodes often occur in an attempt to modulate negative affective states (such as anxious and depressed moods), 
or via impaired impulse control because of the appetite-stimulating effects of high androgen levels. In agreement with the assumption that androgens might trigger binge-purging behavior, findings from a pilot study revealed that the administration of androgen receptor antagonist flutamide leads to a substantial decrease in bulimic symptoms. ${ }^{71}$ Further research is required to provide further support for the different hypotheses presented here.

\section{Metabolic imbalances - insulin resistance}

Most attempts to explain the relationship between PCOS and ED symptomatology, especially binge eating, have rested on the role of insulin. ${ }^{67}$ This has resulted in the proposition of a hyperglycemia hypothesis. Insulin is an important hormone that signals muscle, fat, and liver cells to use glucose from the bloodstream for energy for the body. Almost all women diagnosed with PCOS will have high levels of insulin because of the development of peripheral insulin resistance, which results in weight gain. Insulin resistance is a strong risk factor for metabolic syndrome, a cluster of biochemical and physiological abnormalities associated with the development of cardiovascular disease and type 2 diabetes, which is commonly found in women with PCOS. ${ }^{12}$ Excess weight and inactivity independently increase the severity of insulin resistance, ${ }^{10,62,72}$ and women with PCOS report excess weight as a major source of concern and stress. ${ }^{73} .^{72,74}$

Researchers have made a connection between weight cycling, inflammation, and higher insulin levels. High levels of insulin have been shown to cause low blood sugar, which at times can lead to an appetite stimulant and strong cravings for carbohydrates. ${ }^{34,67}$ The body craves carbohydrates as a physiological need to raise blood sugar to normal levels. ${ }^{36,62,75} \mathrm{~A}$ few studies have found that even a single day of high-fat overfeeding was enough to impair glycemic control and reduce insulin sensitivity in healthy adults. ${ }^{76,77}$ These findings are significant for PCOS patients experiencing binge eating, since they suggest that severe binge eating behaviors may have instantaneous metabolic health consequences for the individual.

These findings have implications for binge eating in PCOS. Altered insulin levels may contribute to weight gain, which is associated with increased distress regarding weight status, initiating the dieting cycle: starting a diet, staying on it for a while with some weight loss, however dietary restraint promotes disinhibition and overeating, leading to binge eating and long-term weight gain through various metabolic and dietary processes, and the cycle repeats. $^{20,23,74}$ However, further research is needed to assess the underlying mechanisms of how insulin may be implicated in the etiology of binge eating and PCOS. ${ }^{76,77}$

\section{Psychological factors}

Many women with PCOS experience symptoms such as acne, hair loss, excessive hair growth, weight gain, which may impact negatively on body image, and subsequently contribute to binge eating behaviors. ${ }^{19,37}$ Research has shown that high levels of body dissatisfaction with selfappearance and physical characteristics are prevalent in women with PCOS (eg, $\left.{ }^{37,78}\right)$. Further, considerable evidence suggests that high levels of body dissatisfaction confer risk for engaging in disordered eating. ${ }^{79}$ For these reasons, individuals with high levels of body dissatisfaction may predispose individuals to overeat or engage in restrictive eating, in an attempt to cope with the psychosocial stressors associated with PCOS symptoms. Such behaviors may lead to clinically significant disordered eating, such as taking laxatives and diet pills, fasting, engaging in excessive exercise, and induced vomiting; culminating in the development of an ED. Moreover, women with PCOS, because of their hormonal imbalances, may be more prone to mood disturbances. ${ }^{19,80}$

Accordingly, recent meta-analyses have revealed an increased prevalence of depression and anxiety associated with PCOS compared with controls. ${ }^{6,16}$ This is significant as negative emotions are a key driver for binge eating behavior, therefore experiencing negative emotions may predispose individuals to engage in disordered eating behaviors to regulate emotions. ${ }^{39}$ Lee and colleagues ${ }^{8}$ results demonstrated that increased ratings of depression and anxiety were related to a higher probability of also presenting with eating pathology and this relationship remained significant even after controlling for age and BMI. Another, large population-based study from Sweden reported an increased probability of PCOS women experiencing BN symptoms, even after controlling for other psychiatric disorders. ${ }^{18}$ The overrepresentation of ED pathology in PCOS populations may be in part explained by the high levels of body dissatisfaction, and increased prevalence of mood and anxiety disorders, which are both independently associated with disordered eating. ${ }^{37,39}$ 


\section{Management of PCOS and binge eating}

Early and reliable identification of binge eating symptoms in PCOS populations is essential for optimal psychological and physical outcomes for women living with PCOS. ${ }^{32}$ However, research has shown that both PCOS and ED diagnoses are often delayed, involve numerous health professionals, and leave women with unmet information needs. ${ }^{73,81}$ Some women describe this time of uncertainty as a physical and "emotional roller-coaster ride". 73 A timely diagnosis of both disorders is therefore important to promote lifestyle management early on to prevent weight gain, obesity, and related metabolic complications. The Androgen Excess-PCOS society guidelines from $2018^{32}$ acknowledge the clinical significance of this issue and advocate for the screening of disordered eating in all women with PCOS. Screening during routine visits can be time-consuming, but measures such as the $\mathrm{SCOFF},{ }^{82}$ comprised of only five questions, could be employed to screen for EDs in routine practice. Furthermore, it is important to raise awareness and provide education to women about disordered eating in PCOS, as many women with EDs are undiagnosed and unaware that their eating and weightrelated thoughts and behaviors are unusual and result in distress. ${ }^{83}$

Addressing the comorbidity of PCOS and EDs provides an exceptional and difficult dilemma of trying to achieve sometimes conflicting goals of weight loss and psychological well-being. Lifestyle modification is the cornerstone of treatment for PCOS women; however, hormonal medication such as insulin sensitizing agents are useful and effective adjuncts to therapy. ${ }^{32}$ Taking a more flexible approach to weight management in women with both PCOS and ED would be optimal in order to prevent accomplishing a "healthy" weight at the cost of quality of psychological well-being. This should ideally be done through a collaborative multidisciplinary effort of numerous health care professionals, including dieticians, mental health professionals, endocrinologists, gynecologists, and primary healthcare providers. It would be important to monitor the PCOS women's diet to help with the symptoms that result from an excess of androgens and insulin resistance, to decrease the risk of diabetes and heart disease later in life. Research has shown that women suffering from PCOS and comorbid mood disorders, benefited more in terms of weight management when undergoing cognitive behavioral therapy (CBT) along with lifestyle changes, than from lifestyle changes alone ${ }^{84}$ Research has also shown that $\mathrm{CBT}^{85}$ and mindfulness ${ }^{86}$ interventions are helpful in reducing food cravings for individuals with BED. However, these treatments have not yet been assessed in PCOS women with comorbid eating pathology, but it is likely that approaching weight management in a way that also tackles the principal psychological mechanisms of EDs, would be more promising than only approaching weight management alone.

\section{Conclusions}

To conclude, the current review has shown that women with PCOS have elevated prevalence and risk factors for binge eating behavior, such as overweight and/or obesity, negative body image, depression and anxiety ${ }^{37,80}$ The suggested links between PCOS and binge eating are not yet well understood, ${ }^{67}$ but it is likely that they may include metabolic (hyperandrogenism), hormonal (insulin resistance), and psychological (body dissatisfaction, depression, anxiety) factors. The interaction of these factors likely is complex in nature. Further research is therefore required to disentangle the intricacies of the relationships between these factors to better comprehend the long-term health risks related to the comorbidity of PCOS and binge eating behaviors. This may be most appropriately achieved through longitudinal studies. Screening PCOS women for binge eating symptoms, and recognition of the life-course implications of the comorbidity of PCOS and binge eating is essential. Identification of the early signs and symptoms for both disorders and collaborative management from patients and health professionals is required to effectively manage PCOS and EDs, and prevent the complications resulting from both disorders. Considering the current array of treatments available for women with PCOS and binge eating, more studies observing the effect of combined treatments for both disorders would be beneficial. The successful management and treatment of PCOS and binge eating behaviors rely on both conditions being addressed in a holistic manner, which ensures all aspects of women with PCOS' functioning are considered prior to the implementation of treatment.

\section{Disclosure}

The authors report no conflicts of interest in this work. 


\section{References}

1. Lizneva D, Suturina L, Walker W, Brakta S, Gavrilova-Jordan L, Azziz R. Criteria, prevalence, and phenotypes of polycystic ovary syndrome. Fertil Steril. 2016;106(1):6-15. doi:10.1016/j.fertnstert.2016.05.003

2. Yildiz BO, Bozdag G, Yapici Z, Esinler I, Yarali H. Prevalence, phenotype and cardiometabolic risk of polycystic ovary syndrome under different diagnostic criteria. Hum Reprod. 2012;27 (10):3067-3073. doi:10.1093/humrep/des232

3. Fr DD, Tarlatzis R. Revised 2003 consensus on diagnostic criteria and long-term health risks related to polycystic ovary syndrome. Fertil Steril. 2004;81(1): 19-25.

4. Moran L, Gibson-Helm M, Teede H, Deeks A. Polycystic ovary syndrome: a biopsychosocial understanding in young women to improve knowledge and treatment options. J Psychosom Obstet Gynecol. 2010;31(1):24-31. doi:10.3109/01674820903477593

5. Hart R, Doherty DA. The potential implications of a PCOS diagnosis on a woman's long-term health using data linkage. J Clin Endocrinol Metab. 2015;100(3):911-919. doi:10.1210/jc.2014-3886

6. Dokras A, Clifton S, Futterweit W, Wild R. Increased prevalence of anxiety symptoms in women with polycystic ovary syndrome: systematic review and meta-analysis. Fertil Steril. 2012;97(1):225-230. e222. doi:10.1016/j.fertnstert.2011.10.022

7. Lee I, Cooney LG, Saini S, Sammel MD, Allison KC, Dokras A. Increased odds of disordered eating in polycystic ovary syndrome: A systematic review and meta-analysis. Eat Weight Disord - Stud Anorexia Bulimia Obesity. 2018. doi:10.1007/s40519-018-0533-y

8. Lee I, Cooney LG, Saini S, et al. Increased risk of disordered eating in polycystic ovary syndrome. Fertil Steril. 2017;107(3):796-802. doi:10.1016/j.fertnstert.2016.12.014

9. Dokras A, Stener-Victorin E, Yildiz BO, et al. Androgen excesspolycystic ovary syndrome society: position statement on depression, anxiety, quality of life, and eating disorders in polycystic ovary syndrome. Fertil Steril. 2018;109(5):888-899. doi:10.1016/j. fertnstert.2018.01.038

10. Norman R, Wu R, Stankiewicz M. Polycystic ovary syndrome. Med J Aust. 2004;180(3):132-137.

11. Teede H, Deeks A, Moran L. Polycystic ovary syndrome: a complex condition with psychological, reproductive and metabolic manifestations that impacts on health across the lifespan. BMC Med. 2010;8:41. doi:10.1186/1741-7015-8-41

12. Lim SS, Kakoly NS, Tan JWJ, et al. Metabolic syndrome in polycystic ovary syndrome: a systematic review, meta-analysis and meta-regression. Obes Rev. 2018. doi:10.1111/obr.12762

13. Moran LJ, Misso ML, Wild RA, Norman RJ. Impaired glucose tolerance, type 2 diabetes and metabolic syndrome in polycystic ovary syndrome: a systematic review and meta-analysis. Hum Reprod Update. 2010;16(4):347-363. doi:10.1093/humupd/dmq001

14. Cooney LG, Dokras A. Beyond fertility: polycystic ovary syndrome and long-term health. Fertil Steril. 2018;110(5):794-809. doi:10.1016/j.fertnstert.2018.08.021

15. Hollinrake E, Abreu A, Maifeld M, Van Voorhis BJ, Dokras A. Increased risk of depressive disorders in women with polycystic ovary syndrome. Fertil Steril. 2007;87(6):1369-1376. doi:10.1016/j. fertnstert.2006.11.039

16. Deeks AA, Gibson-Helm ME, Teede HJ. Anxiety and depression in polycystic ovary syndrome: a comprehensive investigation. Fertil Steril. 2010;93(7):2421-2423. doi:10.1016/j.fertnstert.2009.09.018

17. Annagur BB, Kerimoglu OS, Tazegul A, Gunduz S, Gencoglu BB Psychiatric comorbidity in women with polycystic ovary syndrome. J Obstet Gynaecol Res. 2015;41(8):1229-1233. doi:10.1111/jog.12696

18. Cesta CE, Månsson M, Palm C, Lichtenstein P, Iliadou AN, Landén M. Polycystic ovary syndrome and psychiatric disorders: co-morbidity and heritability in a nationwide Swedish cohort. Psychoneuroendocrinology. 2016;73:196-203. doi:10.1016/j. psyneuen.2016.08.005
19. Himelein MJ, Thatcher SS. Depression and body image among women with polycystic ovary syndrome. J Health Psychol. 2006;11 (4):613-625. doi:10.1177/1359105306065021

20. American Psychiatric Association (APA). Diagnostic and Statistical Manual of Mental Disorders (DSM-5®). Arlington, VA: American Psychiatric Pub; 2013.

21. Montano CB, Rasgon NL, Herman BK. Diagnosing binge eating disorder in a primary care setting. Postgrad Med. 2016;128 (1):115-123. doi:10.1080/00325481.2016.1115330

22. Smink FR, van Hoeken D, Oldehinkel AJ, Hoek HW. Prevalence and severity of DSM-5 eating disorders in a community cohort of adolescents. Int J Eat Disord. 2014;47(6):610-619. doi:10.1002/ eat. 22316

23. Mustelin L, Kaprio J, Keski-Rahkonen A. Beyond the tip of the iceberg: adolescent weight development of women and men with features of binge eating disorder. Eat Behav. 2018;30:83-87. doi:10.1016/j.eatbeh.2018.06.004

24. Lydecker JA, Grilo CM. Comparing men and women with binge-eating disorder and co-morbid obesity. Int $J$ Eat Disord. 2018;51(5):411-417. doi:10.1002/eat.22847

25. Moroshko I, Brennan L, O'Brien P. Predictors of dropout in weight loss interventions: a systematic review of the literature. Obes Rev. 2011;12(11):912-934. doi:10.1111/j.1467-789X.2011.00915.x

26. Grucza RA, Przybeck TR, Cloninger CR. Prevalence and correlates of binge eating disorder in a community sample. Compr Psychiatry. 2007;48(2):124-131. doi:10.1016/j.comppsych.2006.08.002

27. Grilo CM, White MA, Masheb RM. DSM-IV psychiatric disorder comorbidity and its correlates in binge eating disorder. Int $J$ Eat Disord. 2008;42(3):228-234. doi:10.1002/eat.20599

28. Albohn-Kühne C, Rief W. Shame, guilt and social anxiety in obesity with binge-eating disorder. Psychother Psychosom Med Psychol. 2011;61(9-10):412.

29. Cooper PJ, Fairburn CG. Confusion over the core psychopathology of bulimia nervosa. Int J Eat Disord. 1993;13(4):385-389.

30. Sheehan DV, Herman BK. The psychological and medical factors associated with untreated binge eating disorder. Prim Care Companion CNS Disord. 2015;17:2.

31. Da Luz FQ, Hay P, Touyz S, Sainsbury A. Obesity with comorbid eating disorders: associated health risks and treatment approaches. Nutrients. 2018;10(7):829. doi:10.3390/nu10070829

32. Teede HJ, Misso ML, Costello MF, et al. Recommendations from the international evidence-based guideline for the assessment and management of polycystic ovary syndrome. Hum Reprod. 2018;33 (9):1602-1618. doi:10.1093/humrep/dey256

33. Wang R, Mol BWJ. The Rotterdam criteria for polycystic ovary syndrome: evidence-based criteria? Hum Reprod. 2017;32 (2):261-264. doi:10.1093/humrep/dew287

34. Jeanes YM, Reeves S, Gibson EL, Piggott C, May VA, Hart KH. Binge eating behaviours and food cravings in women with polycystic ovary syndrome. Appetite. 2017;109:24-32. doi:10.1016/j. appet.2016.11.010

35. Bernadett M, Szemán-N A. Prevalence of eating disorders among women with polycystic ovary syndrome. Psychiatria Hungarica. 2016;31(2):136-145

36. Larsson I, Hulthén L, Landén M, Pålsson E, Janson P, StenerVictorin E. Dietary intake, resting energy expenditure, and eating behavior in women with and without polycystic ovary syndrome. Clin Nutr. 2016;35(1):213-218. doi:10.1016/j. clnu.2015.02.006

37. Karacan E, Caglar GS, Gürsoy AY, Yilmaz MB. Body satisfaction and eating attitudes among girls and young women with and without polycystic ovary syndrome. J Pediatr Adolesc Gynecol. 2014;27 (2):72-77. doi:10.1016/j.jpag.2013.08.003

38. Batcheller AE, Ressler IB, Sroga JM, Martinez AM, Thomas MA, DiPaola KB. Binge eating disorder in the infertile polycystic ovary syndrome patient. Fertil Steril. 2013;100(3):S413. doi:10.1016/j. fertnstert.2013.07.631 
39. Månsson $\mathrm{M}$, Holte $\mathrm{J}$, Landin-Wilhelmsen $\mathrm{K}$, Dahlgren $\mathrm{E}$, Johansson A, Landén M. Women with polycystic ovary syndrome are often depressed or anxious - a case control study. Psychoneuroendocrinology. 2008;33(8):1132-1138. doi:10.1016/j. psyneuen.2008.06.003

40. Barry DT, Grilo CM, Masheb RM. Comparison of patients with bulimia nervosa, obese patients with binge eating disorder, and nonobese patients with binge eating disorder. J Nerv Ment Dis. 2003;191 (9):589-594. doi:10.1097/01.nmd.0000087185.95446.65

41. Dingemans AE, van Furth EF. Binge eating disorder psychopathology in normal weight and obese individuals. Int $J$ Eat Disord. 2012;45(1):135-138. doi:10.1002/eat.20905

42. McCluskey S, Evans C, Hubert Lacey J, Malcolm Pearce J, Jacobs H. Polycystic ovary syndrome and bulimia *. Fertil Steril. 1991;55 (2):287-291

43. Barr S, Hart K, Reeves S, Sharp K, Jeanes Y. Habitual dietary intake, eating pattern and physical activity of women with polycystic ovary syndrome. Eur J Clin Nutr. 2011;65(10):1126. doi:10.1038/ ejcn.2011.81

44. Michelmore K, Balen A, Dunger D. Polycystic ovaries and eating disorders: are they related? Hum Reprod. 2001;16(4):765-769.

45. Jensterle M, Kocjan T, Kravos NA, Pfeifer M, Janez A. Short-term intervention with liraglutide improved eating behavior in obese women with polycystic ovary syndrome. Endocr Res. 2015;40 (3):133-138. doi:10.3109/07435800.2014.966385

46. Kerchner A, Lester W, Stuart SP, Dokras A. Risk of depression and other mental health disorders in women with polycystic ovary syndrome: a longitudinal study. Fertil Steril. 2009;91(1):207-212. doi:10.1016/j.fertnstert.2007.11.022

47. Wylie J, Barr S, Jeanes Y. Eating frequency and snacking habits in women with polycystic ovary syndrome. J Hum Nutr Diet. 2009;22 (3):274-275. doi:10.1111/jhn.2009.22.issue-3

48. Barry JA, Bouloux P, Hardiman PJ. The impact of eating behavior on psychological symptoms typical of reactive hypoglycemia. A pilot study comparing women with polycystic ovary syndrome to controls. Appetite. 2011;57(1):73-76. doi:10.1016/j.appet.2011.03.003

49. Jahanfar S, Eden J, Nguyent T. Bulimia nervosa and polycystic ovary syndrome. Gynecol Endocrinol. 1995;9(2):113-117.

50. Morgan J, Scholtz S, Lacey H, Conway G. The prevalence of eating disorders in women with facial hirsutism: an epidemiological cohort study. Int J Eat Disord. 2008;41(5):427-431. doi:10.1002/eat.20527

51. Berenson A, Pohlmeier A, Phy J, et al. A low insulinemic diet improves binge eating and quality-of-life in women with PCOS. $J$ Womens Health. 2014;23(4):16.

52. Sbaragli C, Morgante G, Goracci A, Hofkens T, De Leo V, Castrogiovanni P. Infertility and psychiatric morbidity. Fertil Steril. 2008;90(6):2107-2111. doi:10.1016/j.fertnstert.2007.10.045

53. Hay PJ, Mond J, Buttner P, Darby AJ, Murthy RS. Eating disorder behaviors are increasing: findings from two sequential community surveys in South Australia. PLoS One. 2008;3(2):e1541. doi:10.1371/ journal.pone. 0001541

54. Morgan JF, McCluskey SE, Brunton JN, Hubert Lacey J. Polycystic ovarian morphology and bulimia nervosa: a 9-year follow-up study. Fertil Steril. 2002;77(5):928-931.

55. Garner DM, Olmsted MP, Bohr Y, Garfinkel PE. The eating attitudes test: psychometric features and clinical correlates. Psychol Med. 1982;12(4):871-878. doi:10.1017/S0033291700049163

56. Henderson M, Freeman CPL. A self-rating scale for bulimia the 'BITE'. Br J Psychiatry. 1987;150(1):18-24.

57. First MGM, Spitzer R, Williams J. Users Guide for the Structured Clinical Interview for DSM IV Axis I Disorders - Research Version (SCID-I, Version 2.0). New York: New York State Psychiatric Institute; 1996.

58. Fairburn CGCZ, O'Connor M. Eating disorder examination (edition 16.0D). In: Fairburn CG, editor. Cognitive Behavior Therapy and Eating Disorders. New York: Guilford Press; 2008:265-308.
59. Bellver J, Rodriguez-Tabernero L, Robles A, et al. Polycystic ovary syndrome throughout a woman's life. J Assist Reprod Genet. 2018;35 (1):25-39. doi:10.1007/s10815-017-1047-7

60. Klump KL, Keel PK, Racine SE, et al. "The interactive effects of estrogen and progesterone on changes in emotional eating across the menstrual cycle": correction to Klump et al. (2012). J Abnorm Psychol. 2013;122(1):137. doi:10.1037/a0030842

61. Algars M, Huang L, Von Holle AF, et al. Binge eating and menstrual dysfunction. J Psychosom Res. 2014;76(1):19-22. doi:10.1016/j. jpsychores.2013.11.011

62. Maharaj S, Amod A. Polycystic ovary syndrome. J Endocrinol Metab Diabetes S Afr. 2009;14(2):86-95. doi:10.1080/22201009.2009. 10872199

63. Baker JH, Girdler SS, Bulik CM. The role of reproductive hormones in the development and maintenance of eating disorders. Expert Rev Obstet Gynecol. 2012;7(6):573-583. doi:10.1586/eog.12.54

64. Sundblad C, Bergman L, Eriksson E. High levels of free testosterone in women with bulimia nervosa. Acta Psychiatr Scand. 1994;90 (5):397-398.

65. van Anders SM, Watson NV. Social neuroendocrinology: effects of social contexts and behaviors on sex steroids in humans. Hum Nat. 2006;17(2):212-237. doi:10.1007/s12110-006-1018-7

66. Krepula K, Bidzinska-Speichert B, Lenarcik A, TworowskaBardzinska U. Psychiatric disorders related to polycystic ovary syndrome. Endokrynol Pol. 2012;63(6):488-491.

67. Paganini C, Peterson G, Stavropoulos V, Krug I. The overlap between binge eating behaviors and polycystic ovarian syndrome: an etiological integrative model. Curr Pharm Des. 2018;24(9):999-1006. doi:10.2174/1381612824666171204151209

68. Iwasa T, Matsuzaki T, Yano K, et al. Effects of chronic testosterone administration on the degree of preference for a high-fat diet and body weight in gonadal-intact and ovariectomized female rats. Behav Brain Res. 2018;349:102-108. doi:10.1016/j.bbr.2018. 02.021

69. Eriksson E, Sundblad C, Lisjo P, Modigh K, Andersch B. Serum levels of androgens are higher in women with premenstrual irritability and dysphoria than in controls. Psychoneuroendocrinology. 1992;17(2-3):195-204.

70. Ekholm UB, Turkmen S, Hammarback S, Backstrom T, Cooper PJ, Fairburn CG. Sexuality and androgens in women with cyclical mood changes and pre-menstrual syndrome. Acta Obstet Gynecol Scand. 2014;93(3):248-255. doi:10.1111/aogs.12327

71. Sundblad C, Landén M, Eriksson T, Bergman L, Eriksson E. Effects of the androgen antagonist flutamide and the serotonin reuptake inhibitor citalopram in bulimia nervosa: a placebo-controlled pilot study. J Clin Psychopharmacol. 2005;25(1):85-88.

72. Diamanti-Kandarakis E, Papavassiliou AG. Molecular mechanisms of insulin resistance in polycystic ovary syndrome. Trends $\mathrm{Mol} \mathrm{Med}$. 2006;12(7):324-332. doi:10.1016/j.molmed.2006.05.006

73. Gibson-Helm M, Teede H, Dunaif A, Dokras A. Delayed Diagnosis and a lack of information associated with dissatisfaction in women with polycystic ovary syndrome. J Clin Endocrinol Metab. 2017;102 (2):604-612.

74. Neumark-Sztainer D, Wall M, Story M, Standish AR. Dieting and unhealthy weight control behaviors during adolescence: associations with 10-year changes in body mass index. J Adolesc Health. 2012;50 (1):80-86. doi:10.1016/j.jadohealth.2011.05.010

75. Bartholome LT, Raymond NC, Lee SS, Peterson CB, Warren CS. Detailed analysis of binges in obese women with binge eating disorder: comparisons using multiple methods of data collection. Int J Eat Disord. 2006;39(8):685-693. doi:10.1002/(ISSN)1098$108 \mathrm{X}$

76. Parry S, Woods R, Hodson L, Hulston C. A single day of excessive dietary fat intake reduces whole-body insulin sensitivity: the metabolic consequence of binge eating. Nutrients. 2017;9(8):818. doi: $10.3390 /$ nu 9080818 
77. Taylor AE, Hubbard J, Anderson EJ. Impact of binge eating on metabolic and leptin dynamics in normal young women. J Clin Endocrinol Metab. 1999;84(2):428-434. doi:10.1210/jcem.84.2. 5502

78. Kitzinger C, Willmott J. 'The thief of womanhood': women's experience of polycystic ovarian syndrome. Soc Sci Med. 2002;54 (3):349-361. doi:10.1016/S0277-9536(01)00034-X

79. Stice E, Shaw HE. Role of body dissatisfaction in the onset and maintenance of eating pathology: A synthesis of research findings. J Psychosom Res. 2002;53(5):985-993.

80. Cooney LG, Lee I, Sammel MD, Dokras A. High prevalence of moderate and severe depressive and anxiety symptoms in polycystic ovary syndrome: a systematic review and meta-analysis. Hum Reprod. 2017;32(5):1075-1091. doi:10.1093/humrep/dex044

81. Hart LM, Granillo MT, Jorm AF, Paxton S. Unmet need for treatment in the eating disorders: a systematic review of eating disorder specific treatment seeking among community cases. Clin Psychol Rev. 2011;31(5):727-735. doi:10.1016/j.cpr.2011.03.004
82. Morgan JF, Reid F, Lacey JH. The SCOFF questionnaire: a new screening tool for eating disorders. West J Med. 2000;172(3):164-165.

83. Health NIf, Excellence C. Eating Disorders: Recognition and Treatment. UK: NICE; 2017.

84. Cooney L, Milman LW, Sammel M, Allison K, Epperson C, Dokras A. Cognitive behavioral therapy improves weight loss and quality of life in women with polycystic ovary syndrome (PCOS). Fertil Steril. 2016;106 (3):e252-e253. doi:10.1016/j.fertnstert.2016.07.729

85. Amianto F, Ottone L, Abbate Daga G, Fassino S. Binge-eating disorder diagnosis and treatment: a recap in front of DSM-5. BMC Psychiatry. 2015;15(1):70. doi:10.1186/s12888-015-0445-6

86. Godfrey KM, Gallo LC, Afari N. Mindfulness-based interventions for binge eating: a systematic review and meta-analysis. J Behav Med. 2015;38(2):348-362. doi:10.1007/s10865-014-9610-5

\section{Publish your work in this journal}

Neuropsychiatric Disease and Treatment is an international, peerreviewed journal of clinical therapeutics and pharmacology focusing on concise rapid reporting of clinical or pre-clinical studies on a range of neuropsychiatric and neurological disorders. This journal is indexed on PubMed Central, the 'PsycINFO' database and CAS, and is the official journal of The International Neuropsychiatric Association (INA). The manuscript management system is completely online and includes a very quick and fair peer-review system, which is all easy to use. Visit http://www.dovepress.com/testimonials.php to read real quotes from published authors. 